\title{
Remarks on Liouville Type Theorems for Steady-State Navier-Stokes Equations
}

\author{
G Seregin \\ University of Oxford, UK, and PDMI, RAS, Russia
}

October 8, 2018

\begin{abstract}
Liouville type theorems for the stationary Navier-Stokes equations are proven under certain assumptions. These assumptions are motivated by conditions that appear in Liouvile type theorems for the heat equations with a given divergence free drift.
\end{abstract}

\section{Introduction}

Let us consider the classical Navier-Stokes system of partial differential equations describing the steady-state flow of a viscous incompressible fluid in the whole space $\mathbb{R}^{3}$ :

$$
u \cdot \nabla u-\Delta u=-\nabla p, \quad \operatorname{div} u=0 .
$$

Here, $u$ is the three dimensional velocity field, while the pressure $p$ is a scalar function. The classical Liouville type theorem for the stationary NavierStokes equations can be formulated in the following way: prove that any bounded solution $u$ to system (1.1) is constant.

A possible way to attack the above problem is to consider a similar one but for the linear equations with a given divergence free drift, i.e.,

$$
b \cdot \nabla v-\Delta v=-\nabla q, \quad \operatorname{div} v=0, \quad \operatorname{div} b=0,
$$


where $v$ is supposed to be a bounded vector field and $b$ is a divergence free drift satisfying additional conditions.

The linear setup is motivated by the known Liouvile type theorems for an equation

$$
b \cdot \nabla f-\Delta f=0 \quad(\operatorname{div} b=0),
$$

with respect to unknown bounded scalar function $f$ and a given function b. In many cases, the Laplacian in (1.3) can be replaced with an elliptic operator, having measurable coefficients, but it is not the main issue here and we restrict ourselves to the Laplace operator with a drift term only. There are plenty of interesting results related to Liouville type theorems for (1.3) but we are going to mentioned only two of them that will be mimicked in our investigations of (1.1) or (1.2). They are as follows, see [9] and [12] and references there:

Theorem 1.1. Let $f$ be a bounded solutions to (1.3). Assume that either

$$
b \in B M O^{-1}
$$

or

$$
\sup _{1<R<\infty} R^{1-\frac{n}{q}}\|b\|_{q, B(R)}<\infty
$$

for $n / 2<q \leq n$. Then $f$ is identically equal to a constant in $\mathbb{R}^{n}$.

How sharp Theorem 1.1 is unknown, it is a matter of further investigations.

In Theorem 1.1, the $n$-dimensional case is considered. In what follows, it is supposed mostly that $n=3$.

Remark 1.2. In the case $n=3$, by definition, $b \in B M O^{-1}$ if there exists a divergence free vector-valued field $\omega \in B M O$ such that $b=\operatorname{rot} \omega$.

Theorem 1.1 can be proved with the help of Mozer's technique, for example. Although this technique is not applicable to system (1.2), conditions (1.4) and (1.5) may be a good perspective for the steady-state Navier-Stokes equations. As it has been shown in [10] and [11], those conditions appear for the Stokes problem with a drift but with some additional restrictions.

Theorem 1.3. Assume that $v$ is a bounder solution to (1.2) and two additional conditions hold:

$$
\sup _{R>0} R^{\frac{1}{2}-\frac{3}{s}}\|v\|_{s, B(R)}<\infty
$$


with $2 \leq s \leq 6$ and either

$$
b \in B M O^{-1}
$$

or

$$
\sup _{R>0} R^{1-\frac{3}{q}}\|b\|_{L^{q, \infty}(B(R))}<\infty
$$

with $3 / 2<q \leq 3$. Then $v \equiv 0$ in $\mathbb{R}^{3}$.

Here, $\|\cdot\|_{L^{q, \infty}}$ is the norm of the weak Lebesgue space $L^{q, \infty}$.

Comparing with the scalar case, the Liouville type theorem for system (1.2) is true under additional assumption (1.6). The corresponding proof is quite simple and based on the Caccioppoli type inequality and scaling. Our aim is to show that in the case of non-linear system (1.1), the Liouville type theorem is valid for a similar type of conditions as in the scalar case.

Theorem 1.4. Assume that $u$ is a smooth solution to system (1.1) and $u=\operatorname{rot} \omega$ in $\mathbb{R}^{3}$ for a divergence free function $\omega$. Let, for $t>3$ and $\alpha$ subject to

$$
\alpha>-\frac{t-3}{6(t-1)},
$$

the following condition hold:

$$
K_{\alpha}(t):=\sup _{R>0} R^{\alpha}\left(\frac{1}{|B(R)|} \int_{B(R)}\left|\omega-[\omega]_{B(R)}\right|^{t} d x\right)^{\frac{1}{t}}<\infty,
$$

where $[\omega]_{B(R)}$ is the mean value of $\omega$ over the ball $B(R)$. Then $u \equiv 0$ in $\mathbb{R}^{3}$.

Remark 1.5. Conditions (1.10) and (1.9) are satisfied if $\omega$ is a BMOfunction and $\alpha=0$.

Theorem 1.6. Assume that $u$ is a smooth solution to system (1.1) and

$$
M_{\beta}(q):=\sup _{R>0} R^{\beta}\left(\frac{1}{|B(R)|} \int_{B(R)}|u|^{q} d x\right)^{\frac{1}{q}}<\infty
$$

with $3 / 2<q<3$ provided

$$
\beta>\frac{6 q-3}{8 q-6} .
$$

Then $u \equiv 0$ in $\mathbb{R}^{3}$. 
Remark 1.7. For $\beta=1$, the latter condition is similar to (1.5). Indeed, it follows from (1.11) that $M_{1}(q)<\infty$ with $3 / 2<q<3$ implies $u \equiv 0$ in $\mathbb{R}^{3}$.

Theorem 1.6 can be improved for $s>2$.

Theorem 1.8. Assume that $u$ is a smooth solution to system (1.1) and

$$
N_{\gamma}(q):=\sup _{R>0} R^{\gamma-\frac{3}{q}}\|u\|_{L^{q, \infty}}<\infty
$$

with $2<q \leq 3$ provided

$$
\gamma>\frac{4 q-3}{6 q-6}
$$

Then $u \equiv 0$ in $\mathbb{R}^{3}$.

Corollary 1.9. Let $u$ be a bounded smooth solution to (1.1), which is axially symmetric with respect to the axis $x_{3}$, and satisfy the additional assumption $|u(x)| \leq c /\left|x^{\prime}\right|^{\mu}$ for any $x=\left(x^{\prime}, x_{3}\right)$ such that $\left|x^{\prime}\right|>1$, where

$$
\mu>\frac{2}{q_{1}}, \quad q_{1}=\frac{15+\sqrt{33}}{8} .
$$

This result has been already known for $\mu=1$, see [8].

Let us mention another popular Liouville type problem to show that any solution to system (1.1) is identically equal to zero, provided two conditions hold:

$$
\int_{\mathbb{R}^{3}}|\nabla u|^{2} d x<\infty .
$$

and

$$
u(x) \rightarrow 0 \text { as } \quad|x| \rightarrow \infty .
$$

Unfortunately, whether this statement is true or not is still unknown.

One of the best attempts made to solve the above or related problems is presented in [5] where it is shown that the assumption

$$
u \in L_{\frac{9}{2}}\left(\mathbb{R}^{3}\right)
$$

implies $u=0$. Recently, a logarithmic improvement of condition (1.18) has been established in [4]. It is interesting to notice that the statement of Remark 1.9 in general does not follow from (1.18).

More Liouville type results can be found in interesting papers [7], [8], [3], and 2] and references there. 


\section{Proof of Main Result}

\subsection{Caccioppoli Type Inequality}

In the first part of the proof, we are going to use similar arguments as in [10] with some changes. We fix $R>0$ arbitrarily and take a non-negative cut-off function $\varphi \in C_{0}^{\infty}(B(R))$ with the following properties: $\varphi(x)=1$ in $B(\varrho)$, $\varphi(x)=0$ out of $B(r)$, and $\left|\nabla^{k} \varphi(x)\right| \leq c /(r-\varrho)^{k}$ for any $R / 2 \leq \varrho<r \leq R$ and $k=1,2,3,4$.

Assume also that we are given two divergence free functions $\bar{\omega}$ and $\widetilde{\omega}$ such that $u=\operatorname{rot} \bar{\omega}=\operatorname{rot} \widetilde{\omega}$ in $B(R)$.

For a given $2<s<\infty$, there exists a constant $c_{0}=c_{0}(s)>0$ and a function $w \in W_{s}^{1}(B(r))$, vanishing on $\partial B(r)$, such that $\operatorname{div} w=\nabla \varphi \cdot u$ and

$$
\int_{B(r)}|\nabla w|^{t} d x \leq c_{0}(s) \int_{B(r)}|\nabla \varphi \cdot u|^{t} d x
$$

for $t=2$ and for $t=s$.

Now, we let us test the Navier-Stokes equations (1.1) with the function $\varphi u-w$. After integration by parts over $B(r)$, we find the following identity

$$
\begin{aligned}
& \int_{B(r)} \varphi|\nabla u|^{2} d x=-\int_{B(r)} \nabla u:(\nabla \varphi \otimes u) d x+\int_{B(r)} \nabla w: \nabla u d x+ \\
& -\int_{B(r)}(u \cdot \nabla u) \cdot \varphi u d x+\int_{B(r)}(u \cdot \nabla u) \cdot w d x=I_{1}+I_{2}+I_{3}+I_{4} .
\end{aligned}
$$

The first two terms can be estimated easily. As a result,

$$
\left|I_{1}\right|+\left|I_{2}\right| \leq c\left(\int_{B(r)}|\nabla u|^{2} d x\right)^{\frac{1}{2}}\left(\int_{B(r)}|\nabla \varphi|^{2}|u|^{2} d x\right)^{\frac{1}{2}}
$$

To estimate $I_{3}$ and $I_{4}$, we are going to use integration by parts and elementary properties of the differential operator rot. Indeed, we have

$$
\left|I_{3}\right|=\left|\int_{B(r)} u_{j} u_{i, j} u_{i} \varphi d x\right|=\left.\frac{1}{2}\left|\int_{B(r)} \operatorname{rot} \bar{\omega} \cdot \nabla\right| u\right|^{2} \varphi d x\left|=\frac{1}{2}\right| \int_{B(r)} \bar{\omega} \cdot\left(\nabla|u|^{2} \times \nabla \varphi\right) d x \mid
$$




$$
\begin{gathered}
\leq\left(\int_{B(r)}|\nabla u|^{2} d x\right)^{\frac{1}{2}}\left(\int_{B(r)}|\nabla \varphi|^{2}|\bar{\omega}|^{2}|u|^{2} d x\right)^{\frac{1}{2}} \leq \\
\leq\left(\int_{B(r)}|\nabla u|^{2} d x\right)^{\frac{1}{2}}\left(\int_{B(r)}|\nabla \varphi|^{s}|u|^{s} d x\right)^{\frac{1}{s}}\left(\int_{B(r)}|\bar{\omega}|^{\frac{2 s}{s-2}} d x\right)^{\frac{s-2}{2 s}} .
\end{gathered}
$$

It remains to evaluated $I_{4}$ :

$$
\begin{gathered}
\left|I_{4}\right|=\left|\int_{B(r)}(\operatorname{rot} \bar{\omega})_{j} u_{k, j} w_{k} d x\right|=\left|\int_{B(r)} \bar{\omega} \cdot\left(\nabla u_{k} \times \nabla w_{k}\right) d x\right| \leq \\
\leq\left(\int_{B(r)}|\nabla u|^{2} d x\right)^{\frac{1}{2}}\left(\int_{B(r)}|\bar{\omega}|^{2}|\nabla w|^{2} d x\right)^{\frac{1}{2}} \leq \\
\leq\left(\int_{B(r)}|\nabla u|^{2} d x\right)^{\frac{1}{2}}\left(\int_{B(r)}|\nabla w|^{s} d x\right)^{\frac{1}{s}}\left(\int_{B(r)}|\bar{\omega}|^{\frac{2 s}{s-2}} d x\right)^{\frac{s-2}{2 s}} \leq \\
\leq c(s)\left(\int_{B(r)}|\nabla u|^{2} d x\right)^{\frac{1}{2}}\left(\int_{B(r)}|\nabla \varphi|^{s}|u|^{s} d x\right)^{\frac{1}{s}}\left(\int_{B(r)}|\bar{\omega}|^{\frac{2 s}{s-2}} d x\right)^{\frac{s-2}{2 s}} .
\end{gathered}
$$

So, after application of Young inequality, we arrive at the following estimate

$$
\begin{aligned}
& \int_{B(\varrho)}|\nabla u|^{2} d x \leq \frac{1}{8} \int_{B(r)}|\nabla u|^{2} d x+c \int_{B(r)}|\nabla \varphi|^{2}|u|^{2} d x+ \\
& \quad+c(s)\left(\int_{B(r)}|\nabla \varphi|^{s}|u|^{s} d x\right)^{\frac{2}{s}}\left(\int_{B(r)}|\bar{\omega}|^{\frac{2 s}{s-2}} d x\right)^{\frac{s-2}{s}} .
\end{aligned}
$$

Our next step is the application of a multiplicative inequality

$$
\begin{aligned}
\left(\int_{B(r)}|\nabla \varphi|^{s}|u|^{s} d x\right)^{\frac{2}{s}} \leq & c\left(\int_{B(r)}|\nabla \varphi|^{2}|u|^{2} d x\right)^{1-\lambda}\left(\left(\int_{B(r)}|\nabla \varphi|^{2}|\nabla u|^{2} d x\right)^{\lambda}+\right. \\
& \left.+\left(\left.\int_{B(r)}|\nabla| \nabla \varphi\right|^{2}|u|^{2} d x\right)^{\lambda}\right)
\end{aligned}
$$


with $\lambda=3 \frac{s-2}{2 s}$. It is legal under the additional assumption $s<6$. Applying the Young inequality one more time, we find

$$
\begin{gathered}
\int_{B(\varrho)}|\nabla u|^{2} d x \leq \frac{1}{4} \int_{B(r)}|\nabla u|^{2} d x+c \int_{B(r)}|\nabla \varphi|^{2}|u|^{2} d x+ \\
+c(s)\left(\int_{B(r)}|\nabla \varphi|^{2}|u|^{2} d x\right)^{1-\lambda}\left(\left.\int_{B(r)}|\nabla| \nabla \varphi\right|^{2}|u|^{2} d x\right)^{\lambda}\left(\int_{B(r)}|\bar{\omega}|^{\frac{2 s}{s-2}} d x\right)^{\frac{s-2}{s}}+ \\
+c(s)\left(\frac{1}{(r-\varphi)^{2}}\right)^{\frac{\lambda}{1-\lambda}} \int_{B(r)}|\nabla \varphi|^{2}|u|^{2} d x\left(\int_{B(r)}|\bar{\omega}|^{\frac{2 s}{s-2}} d x\right)^{\frac{s-2}{s(1-\lambda)}} .
\end{gathered}
$$

To proceed further, we need the following auxiliary lemma.

Lemma 2.1. Let $\psi$ be a bounded non-negative twice differentiable functions with compact support in $B(R)$. Then

$$
\int \psi|u|^{2} d x \leq c\left(\int \psi|\nabla u|^{2} d x\right)^{\frac{1}{2}}\left(\int \psi|\widetilde{\omega}|^{2} d x\right)^{\frac{1}{2}}+c \int\left|\nabla^{2} \psi\right|^{2}|\widetilde{\omega}|^{2} d x .
$$

Proof of Lemma 2.1 is based on the elementary identity:

$$
\int \psi|\operatorname{rot} \widetilde{\omega}|^{2} d x=\int \psi \operatorname{rot} \operatorname{rot} \widetilde{\omega} \cdot \widetilde{\omega} d x-\int \nabla^{2} \psi: \widetilde{\omega} \otimes \widetilde{\omega} d x+\frac{1}{2} \int \Delta \psi|\widetilde{\omega}|^{2} d x .
$$

It follows from Lemma 2.1 that

$$
\begin{gathered}
\int_{B(r)}|\nabla \varphi|^{2}|u|^{2} d x \leq \\
\leq \frac{c}{(r-\varrho)^{2}}\left[\left(\int_{B(r) \backslash B(\varrho)}|\widetilde{\omega}|^{2} d x\right)^{\frac{1}{2}}\left(\int_{B(r) \backslash B(\varrho)}|\nabla u|^{2} d x\right)^{\frac{1}{2}}+\frac{1}{(r-\varrho)^{2}} \int_{B(r) \backslash B(\varrho)}|\widetilde{\omega}|^{2} d x\right]
\end{gathered}
$$

and

$$
\begin{gathered}
\int_{B(r)}\left|\nabla^{2} \varphi\right|^{2}|u|^{2} d x \leq \\
\leq \frac{c}{(r-\varrho)^{4}}\left[\left(\int_{B(r) \backslash B(\varrho)}|\widetilde{\omega}|^{2} d x\right)^{\frac{1}{2}}\left(\int_{B(r) \backslash B(\varrho)}|\nabla u|^{2} d x\right)^{\frac{1}{2}}+\frac{1}{(r-\varrho)^{2}} \int_{B(r) \backslash B(\varrho)}|\widetilde{\omega}|^{2} d x\right] .
\end{gathered}
$$


So, the main inequality can be transformed to the following form

$$
\begin{gathered}
\int_{B(\varrho)}|\nabla u|^{2} d x \leq \frac{1}{4} \int_{B(r)}|\nabla u|^{2} d x+ \\
+\frac{c}{(r-\varrho)^{2}}\left[\left(\int_{B(r) \backslash B(\varrho)}|\widetilde{\omega}|^{2} d x\right)^{\frac{1}{2}}\left(\int_{B(r) \backslash B(\varrho)}|\nabla u|^{2} d x\right)^{\frac{1}{2}}+\frac{1}{(r-\varrho)^{2}} \int_{B(r) \backslash B(\varrho)}|\widetilde{\omega}|^{2} d x\right] \times \\
\times\left(1+\left(\frac{1}{(r-\varrho)^{3}} \int_{B(r)}|\bar{\omega}|^{\frac{2 s}{s-2}} d x\right)^{2 \frac{s-2}{6-s}}+\left(\frac{1}{(r-\varrho)^{3}} \int_{B(r)}|\bar{\omega}|^{\frac{2 s}{s-2}} d x\right)^{\frac{s-2}{s}}\right) .
\end{gathered}
$$

Now, we are going to apply the Young inequality and find

$$
\begin{gathered}
\int_{B(\varrho)}|\nabla u|^{2} d x \leq \frac{1}{2} \int_{B(r)}|\nabla u|^{2} d x+ \\
+\frac{c}{(r-\varrho)^{4}} \int_{B(r) \backslash B(\varrho)}|\widetilde{\omega}|^{2} d x \times \\
\times\left(1+\left(\frac{1}{(r-\varrho)^{3}} \int_{B(r)}|\bar{\omega}|^{\frac{2 s}{s-2}} d x\right)^{2 \frac{s-2}{6-s}}+\left(\frac{1}{(r-\varrho)^{3}} \int_{B(r)}|\bar{\omega}|^{\frac{2 s}{s-2}} d x\right)^{\frac{s-2}{s}}\right)^{2} \leq \\
\leq \frac{1}{c} \int_{B(r)}|\nabla u|^{2} d x+\int_{B(r) \backslash B(\varrho)}|\widetilde{\omega}|^{2}\left(1+\left(\frac{1}{(r-\varrho)^{3}} \int_{B(r)}|\bar{\omega}|^{\frac{2 s}{s-2}} d x\right)^{4 \frac{s-2}{6-s}}\right) .
\end{gathered}
$$

Using known iterative arguments, see [6], we can deduce from the latter inequality the following:

$$
\begin{gathered}
\int_{B(R / 2)}|\nabla u|^{2} d x \leq \frac{c}{R}\left(\frac{1}{|B(R)|} \int_{B(R) \backslash B(R / 2)}|\widetilde{\omega}|^{2} d x\right)(1+ \\
\left.+\left(\frac{1}{|B(R)|} \int_{B(R)}|\bar{\omega}|^{\frac{2 s}{s-2}} d x\right)^{4 \frac{s-2}{6-s}}\right) .
\end{gathered}
$$


Now, after the change

$$
t=\frac{2 s}{s-2},
$$

we find

$$
\begin{aligned}
\int_{B(R / 2)} \mid & \left.\nabla u\right|^{2} d x \leq \frac{c}{R}\left(\frac{1}{|B(R)|} \int_{B(R) \backslash B(R / 2)}|\widetilde{\omega}|^{2} d x\right) \times \\
& \times\left(1+\left(\frac{1}{|B(R)|} \int_{B(R)}|\bar{\omega}|^{t} d x\right)^{\frac{4}{t-3}}\right),
\end{aligned}
$$

with the range for $t>3$.

Obviously, Caccioppoli's type inequality (2.1) is going to be the main sought for proving Liouville type theorems. Indeed, we need to find reason-

able conditions under which the right hand side of (2.1) tends to zero as $R \rightarrow \infty$.

\subsection{Proof of Theorem 1.4}

Here, we let

$$
\bar{\omega}=\widetilde{\omega}=\omega-[\omega]_{B(R)}
$$

and assume that and conditions (1.10) and (1.9) hold. Then (2.1) implies

$$
\begin{gathered}
\int_{B(R / 2)}|\nabla u|^{2} d x \leq \frac{c}{R}\left(\frac{1}{|B(R)|} \int_{B(R)}|\bar{\omega}|^{2} d x\right) \times \\
\times\left(1+\left(\frac{1}{|B(R)|} \int_{B(R)}|\bar{\omega}|^{t} d x\right)^{\frac{4}{t-3}}\right),
\end{gathered}
$$

with the right hand side tending to zero as $R \rightarrow \infty$.

\subsection{Proof of Theorem 1.6}

Here, we let

$$
\bar{\omega}=\widetilde{\omega}=\omega^{R},
$$

where $\omega^{R}$ is a unique solution the following boundary value problem

$$
\operatorname{rot} \omega^{R}=u, \quad \operatorname{div} \omega^{R}=0
$$


in $B(R)$ and

$$
\omega^{R} \cdot \nu=0
$$

on $\partial B(R)$, where $\nu$ is a unit outward normal to $\partial B(R)$. Then (2.1) implies

$$
\begin{gathered}
\int_{B(R / 2)}|\nabla u|^{2} d x \leq \frac{c}{R}\left(\frac{1}{|B(R)|} \int_{B(R)}\left|\omega^{R}\right|^{2} d x\right) \times \\
\times\left(1+\left(\frac{1}{|B(R)|} \int_{B(R)}\left|\omega^{R}\right|^{t} d x\right)^{\frac{4}{t-3}}\right),
\end{gathered}
$$

By scaling, we have the following inequalities, see [1],

$$
\int_{B(R)}\left|\omega^{R}\right|^{2} d x \leq c R^{2}\left(\int_{B(R)}\left|\operatorname{rot} \omega^{R}\right|^{q} d x\right)^{\frac{2}{q}} R^{3\left(1-\frac{2}{q}\right)}
$$

and

$$
\left(\int_{B(R)}\left|\omega^{R}\right|^{\frac{3 q}{3-q}} d x\right)^{\frac{3-q}{3 q}} \leq\left(\int_{B(R)}\left|\operatorname{rot} \omega^{R}\right|^{q} d x\right)^{\frac{1}{q}}
$$

Next, letting $t=3 q /(3-q)$, we can re-write (2.3) so that

$$
\begin{gathered}
\int_{B(R / 2)}|\nabla u|^{2} d x \leq c R\left(\frac{1}{|B(R)|} \int_{B(R)}|u|^{q} d x\right)^{\frac{2}{q}} \times \\
\times\left(1+R^{\frac{4 q}{2 q-3}}\left(\frac{1}{|B(R)|} \int_{B(R)}|u|^{q} q x\right)^{\frac{4}{2 q-3}}\right) .
\end{gathered}
$$

Letting $t=3 q /(3-q)$, we have

$$
\int_{B(R / 2)}|\nabla u|^{2} d x \leq c M_{\beta}(q)\left[R^{1-2 \beta}+\left(M_{\beta}(q)\right)^{\frac{4 q}{2 q-3}} R^{\frac{4 q}{2 q-3}(1-\beta)+(1-2 \beta)}\right] \rightarrow 0
$$

as $R \rightarrow \infty$ provided (1.12) holds. 


\subsection{Proof of Theorem 1.8}

Repeating arguments from ([1] $)$, we have the following estimate

$$
\int_{B(R / 2)}|\nabla u|^{2} d x \leq \frac{c}{R^{2}} \int_{B(R)}\left|u-[u]_{B(R)}\right|^{2} d x\left[1+\left(R^{1-\frac{3}{q}}\|u\|_{L^{q, \infty}}\right)^{\frac{2 q}{2 q-3}}\right] .
$$

After application of Hölder inequality, we find

$$
\int_{B(R / 2)}|\nabla u|^{2} d x \leq \frac{c}{R}\left(N_{\gamma}(q) R^{1-\gamma}\right)^{2}\left[1+\left(N_{\gamma}(q) R^{1-\gamma}\right)^{\frac{2 q}{2 q-3}}\right] .
$$

The right hand side of the latter inequality tends to zero as $R \rightarrow \infty$ provided (1.14) holds.

\subsection{Proof of Corollary 1.9}

Here, we are going to replace balls $B(R)$ with cylinders $C(R)=b(R) \times]-$ $R, R[$, where $b(R)$ is the two-dimensional ball of radius $R$ centred at the origin. We also can replace the weak Lebesgue space $L^{q, \infty}(B(R))$ with the usual Lebesgue space $L_{q}(B(R))$. Without loss of generality, we may assume that $|u| \leq 1$.

Assume that condition (1.15) holds. We can find $q<q_{1}$ so that $\mu>$ $2 / q>2 / q_{1}$. This allows us to set $\gamma=\frac{2}{q}$. By the choice of $q$, we have

$$
\gamma>\frac{4 q-3}{6 q-6}
$$

and thus restriction (1.14) is fulfilled.

Next, it is obvious that $N_{\gamma}(q)=\max \left\{I_{1}, I_{2}\right\}$, where

$$
I_{1}=\sup _{0<R \leq 1} R^{-\frac{1}{q}}\left(\int_{C(R)}|u|^{q} d x\right)^{\frac{1}{q}} \leq c<\infty
$$

and

$$
\sup _{R>1} R^{-\frac{1}{q}}\left(\int_{C(R)}|u|^{q} d x\right)^{\frac{1}{q}} \leq J_{1}+J_{2}
$$


Here,

$$
\begin{gathered}
J_{1}=\sup _{R>1} R^{-\frac{1}{q}}\left(\int_{b(1) \times]-R, R[}|u|^{q} d x\right)^{\frac{1}{q}}, \\
J_{2}=\sup _{R>1} R^{-\frac{1}{q}}\left(\int_{(b(R) \backslash b(1)) \times]-R, R[}|u|^{q} d x\right)^{\frac{1}{q}} .
\end{gathered}
$$

Introducing polar coordinates, we observe that $J_{1}$ is bounded and

$$
J_{2} \leq c \sup _{R>1} R^{-\frac{1}{q}}\left(2 R \int_{1}^{R} \varrho^{1-\mu q} d \varrho\right)^{\frac{1}{q}} \leq c \sup _{R>1} R^{-\frac{1}{q}}\left(2 R\left(1-R^{2-\mu q}\right)\right)^{\frac{1}{q}} \leq c .
$$

\section{References}

[1] Bourguignon, J.P., Brezis, H., Remarks on Euler Equation, J. Funct. Anal., 15, 341-363 (1974).

[2] Chae, D., Liouville-Type Theorem for the Forced Euler Equations and the Navier-Stokes Equations. Commun. Math. Phys.326: 37-48 (2014).

[3] Chae, D., Yoneda, T., On the Liouville theorem for the stationary Navier-Stokes equations in a critical space, J. Math. Anal. Appl. 405 (2013), no. 2, 706-710.

[4] Chae, G., Wolf, J., On Liouville type theorems for the steady NavierStokes equations in $R^{3}$, arXiv:1604.07643.

[5] Galdi, G. P. An introduction to the mathematical theory of the NavierStokes equations. Steady-state problems. Second edition. Springer Monographs in Mathematics. Springer, New York, 2011. xiv+1018 pp.

[6] Giaquinta, M., Multiple integrals in the calculus of variations and nonlinear elliptic systems. Annals of Mathematics Studies, 105. Princeton University Press, Princeton, NJ, 1983. vii+297 pp.

[7] Gilbarg, D., Weinberger, H. F. Asymptotic properties of steady plane solutions of the Navier- Stokes equations with bounded Dirichlet integral, Ann. Scuola Norm. Sup. Pisa Cl. Sci.(4) 5 (1978), no. 2, 381-404. 
[8] Koch, G., Nadirashvili, N., Seregin, G., Sverak, V., Liouville theorems for the Navier-Stokes equations and applications, Acta Mathematica, 203 (2009), 83-105.

[9] Nazarov, A. I., Uraltseva, N. N., The Harnack inequality and related properties for solutions to elliptic and parabolic equations with divergence-free lower-order coefficients, St. Petersburg Mathematical Journal, 2012, 23:1, 93-115.

[10] Seregin, G., Liouville type theorem for stationary Navier-Stokes equations, Nonlinearity, 29 (2016), 2191-2195.

[11] Seregin, G., A Liouville Type Theorem for Steady-State Navier-Stokes Equations, arXiv:1611.01563

[12] Seregin, G., Silvestre, L., Sverak, V., Zlatos, A., On divergence-free drifts, Journal of Differential Equations, Volume 252, Issue 1, January 2012, pp. 505-540.

[13] Stein, Elias M. Singular integrals and differentiability properties of functions. Princeton Mathematical Series, No. 30 Princeton University Press, Princeton, N.J. 1970 xiv+290 pp. 